\title{
Application Research of Network Learning Algorithm Based on Neural Network Dis- turbance Compensation in Satellite Attitude Control
}

\author{
F.Leo John ${ }^{1}$, Deeksha Dogra ${ }^{1,2^{*}}$ \\ 1. Independent Researcher and Research Consultant, Tamil Nadu, India \\ 2. Shoolini University of Biotechnology and Management Sciences, School of Biological \\ and Environmental Sciences, Solan - 173229, Himachal Pradesh, India.
}

\author{
Email: drleojohn@yahoo.com \\ deekshadogra80@gmail.com
}

\begin{abstract}
Based on the satellite attitude control method, this paper proposes an attitude control method based on neural network disturbance compensation. The paper firstly analyzes the neural network algorithm and proposes an orthogonal least squares algorithm to implement network learning. In this paper, a set of high-precision directional neural network compensation controllers is designed for the attitude control of acupuncture small satellites. The feasibility of the improved orthogonal least squares algorithm combined with the neural network supplementary control method in satellite attitude control is verified by experiments.
\end{abstract}

Keywords: orthogonal least squares algorithm, neural network, disturbance compensation; satellite attitude control

\section{Introduction}

It is used for surveying, reconnaissance and other satellites that require highprecision orientation. The attitude accuracy will be seriously affected by various disturbances. The traditional methods cannot provide satisfactory control measures, and lack of adaptive ability and robustness. Modern aircraft often have strong coupling, fast time-varying, and nonlinear characteristics. Traditional modeling and control methods have been increasingly unable to meet the needs of controller design. Traditional intelligent control based on expert system has the "bottleneck" problem of knowledge acquisition; feedback linearization, differential geometry and other methods can only solve limited nonlinear problems, and lack of universality. Because neural network has the advantages of parallelism, rapidity, fault tolerance and associative memory when processing information, and has good ability to approximate nonlinear functions, it has been widely recognized and extensively studied by experts and scholars in various fields, and 
has obtained a large number of Research results. The data elaborated by Habibkhan and Arasi et al. (2017) the orthogonal least squares algorithm and its improved normative orthogonal least squares algorithm are proposed for RBF neural networks, which simplifies the network structure and improves the network learning effect.

However, it is worth noting that most of the research results are focused on using neural networks to build inverse models of systems or directly acting as controllers. Research on modeling disturbances directly and eliminating the effects of disturbances is rare. The literature of Steyn et al. (2015) was studied the neural network compensation problem of servo system disturbance torque. In this paper, the satellite attitude control system is introduced into the neural network to model the disturbance torque, and then the dynamic compensation of the disturbance torque is realized according to the full compensation principle to improve the performance of the satellite attitude control system.

\section{Neural network model}

\subsection{Modeling of the GM $(1,1)$ model}

The prediction method and the traditional statistical prediction method have many advantages. It does not need to determine whether the predictor conforms to the normal distribution, does not require large sample statistic, does not need to change the prediction model at any time according to the change of the input variable, and generates by accumulating Fan et al. (2015) The technique establishes a unified differential equation model, and after cumulative reduction, the prediction result is obtained, and the differential equation model has higher prediction accuracy. The essence of establishing the GM $(1,1)$ model is to accumulate the original data once, so that the generated series exhibits a certain regularity. By establishing a differential equation model, the fitting curve is obtained to predict the system by Shaofei (2020).

Time series $x^{(0)}=\left(x^{(0)}(1), x^{(0)}(2), \ldots \ldots, x^{(0)}(n)\right)$ is used for first-order accumulation to generate 1-AGO:

$$
\begin{aligned}
& x^{(I)}=\left\{x^{(I)}(1), x^{(l)}(2), x^{(I)}(3), \ldots, x^{(I)}(n)\right\} \\
& x^{(I)}(k)=\sum_{i=0}^{k}\left(x^{(0)}(k)\right)
\end{aligned}
$$

After constructing the first-order linear differential equation, the whitening differential equation of the equation is:

$$
\frac{d x^{(1)}}{d t}+a x^{(1)}=u
$$

Using the least squares method to solve a, u have

$$
\alpha=\left[\begin{array}{l}
a \\
u
\end{array}\right]=\left(B^{T} B\right)^{-1} B^{T} Y_{N}
$$

In the middle

$$
B=\left[\begin{array}{cc}
-0.5\left(x^{(1)}(2)+x^{(1)}(1)\right) & 1 \\
-0.5\left(x^{(1)}(3)+x^{(1)}(2)\right) & 1 \\
\vdots & \vdots \\
-0.5\left(x^{(1)}(n)+x^{(1)}(n-1)\right) & 1
\end{array}\right] \quad Y_{N}=\left[\begin{array}{c}
x^{(0)}(2) \\
x^{(0)}(3) \\
\vdots \\
x^{(0)}(n)
\end{array}\right]
$$

The prediction model of $x^{(1)}$ is

$$
\hat{x}^{(1)}(k+1)=\left(x^{(0)}(1)-\frac{u}{a}\right) e^{-a k}+\frac{u}{a}
$$

The prediction model of $x^{(0)}$ is

$$
\hat{x}^{(0)}(k+1)=\left(1-e^{a}\right)\left(x^{(0)}(1)-\frac{u}{a}\right) e^{-a k},(k=1,2, \cdots,)
$$

\section{2. $G M(1,1)$ model residuals and relative residuals}

Set the original discrete non-negative column $X^{(0)}$ :

$$
X^{(o)}=\left\{x^{(0)}(1), x^{(0)}(2), x^{(o)}(3), \ldots, x^{(0)}(n)\right\}
$$


Sequence $\hat{X}^{(0)}$ is the fitted sequence after solving the GM $(1,1)$ model:

$$
\hat{X}^{(o)}=\left\{\hat{x}^{(0)}(1), \hat{x}^{(o)}(2), \hat{x}^{(0)}(3), \ldots, \hat{x}^{(o)}(n)\right\}
$$

Residual number $\varepsilon^{(0)}$ :

$$
\varepsilon^{(0)}=\left\{x^{(o)}(1)-\hat{x}^{(0)}(1), \ldots, x^{(o)}(n)-\hat{x}^{(o)}(n)\right\}
$$

The relative residual sequence $\Delta$ is expressed as:

$$
\Delta=\left\{\left|\frac{\varepsilon^{(l)}}{x^{(o)}(1)}\right|,\left|\frac{\varepsilon^{(2)}}{x^{(o)}(2)}\right|, \ldots,\left|\frac{\varepsilon^{(n)}}{x^{(o)}(n)}\right|\right\}
$$

\subsection{Neural network combination model}

The neural network model is a complete fusion of the method and the neural network, so the model has both the advantages of the system and the neural network. The modeling idea of the neural network is: firstly, the $\operatorname{GM}(1,1)$ model is established for each of the plurality of series, and a series of predicted values of the original sequence data can be obtained, and the predicted values may have a certain deviation from the original data, and this is more There is also a certain correlation between the original sequences, and this relationship is not very clear. The deviation between these predicted values and actual values and the relationship between the sequences can be integrated into the neural network model. The predicted value formed by $\operatorname{GM}(1,1)$ is used as the input sample of the neural network, and the actual value is used as the nerve. The output samples of the network, taking a certain structure, and then training the network, can obtain a series of weights and thresholds corresponding to the corresponding nodes. The predicted values of the GM $(1,1)$ models for the next time or multiple times are used as the input of the neural network, and the corresponding output is obtained as the final predicted value at the next time or multiple times (Shirazi et al. (2016).
The neural network mainly passes through a three-layer BP neural network: with deviation and at least one S-type hidden layer plus a linear output layer can approximate the characteristics of any rational function, through training to make the neural network to simulate the sequence data between and between the sequence's relationship. Suppose there are $\mathrm{m}$ interrelated series, and each series has $\mathrm{n}$ data as that elaborated by Wang et al. (2015).

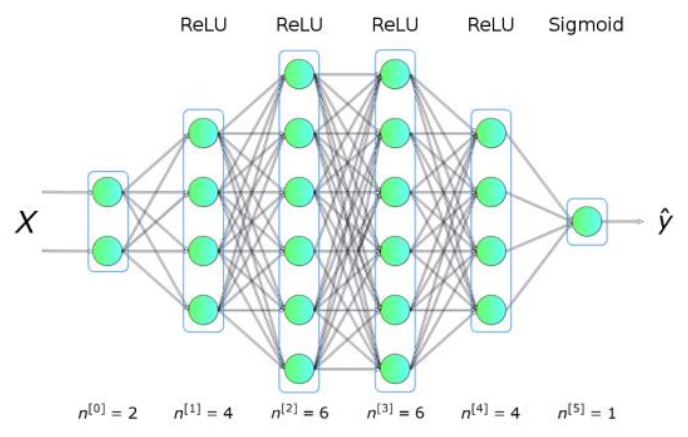

Fig. 1. The basic principle of neural network algorithm.

\section{Orthogonal least squares algorithm}

\subsection{Proposal of the problem}

$\mathrm{RBF}$ network learning algorithm There are four parameters to be learned in the RBF network, namely the number of hidden layer nodes, the center of the basis function, the width of the basis function, and the connection weight of the network hidden layer to the output layer. However, the influence of the form of the nonlinear function and the width of the basis function used in the RBF network on the network performance is not critical. The key factor is the selection of the center of the basis function. The performance of the RBF network constructed by the center is not optimal. Satisfied. The shape pattern recognition model is estab- 
lished by orthogonal least squares algorithm. The orthogonal least squares algorithm is currently a method for training RBF networks. This method is simple and easy to operate.

\subsection{Solving the problem}

The optimal number of neurons in the hidden layer of the network and the center of the hidden layer basis function can be determined by the orthogonal optimization method. Finally, the connection weight of the network hidden layer to the output layer is obtained by the linear optimization algorithm. We use the orthogonal least squares algorithm to train the RBF network. We start to analyze the algorithm below. Let the number of network learning samples be $\mathrm{N}$, and the learning sample input and output be $\{D(k), Y(k)\}_{k-1}^{N}$ and $D(k)=\left[d_{1}(k), d_{2}(k), \ldots, d_{n i}(k)\right]$. The number of initial neurons in the network hidden layer is selected as $\mathrm{N}$, and the center of the hidden layer basis function is selected from the learning samples.

The expected output of the RBF network can be expressed by (10):

$$
Y=P \theta+E
$$

Among them:

$$
\begin{aligned}
& Y=\left[y_{1}, y_{2}, \ldots, y_{n o}\right] \\
& y_{i}=\left[y_{i}(1), y_{i}(2), \ldots, y_{i}(N)\right]^{T} \\
& P=\left[p_{1}, p_{2}, \ldots, p_{N}\right] \\
& p_{i}=\left[p_{i}(1), p_{i}(2), \ldots, p_{i}(N)\right]^{T}, \quad 1 \leq i \leq N \\
& \theta=\left[\theta_{1}, \theta_{2}, \ldots, \theta_{n 0}\right], \theta_{i}=\left[\theta_{1 i}, \theta_{2 i}, \ldots, \theta_{N i}\right]^{T}, 1 \leq i \leq n_{0} \\
& E=\left[e_{1}, e_{2}, \ldots, e_{n 0}\right], e_{i}=\left[e_{i}(1), e_{i}(2), \ldots, e_{i}(N)\right]^{T}, 1 \leq i \leq n_{0}
\end{aligned}
$$

$\mathrm{P}$ is the hidden layer output matrix, $\theta$ is the connection weight matrix from the hidden layer to the output layer, and $E$ is the error matrix between the expected output of the network and the actual output.

The RBF network can be regarded as a special case of the linear regression model
(10), and the regression amount $p_{i}$ constitutes a set of base vectors. Since the width of the basis function has little effect on the network performance, we assume that the width of the basis function is constant, so that the center of a basis function corresponds to a regression amount $p_{i}$. Therefore, how to select the center of the RBF network hidden layer basis function from the input samples the problem is transformed into the problem of selecting important regressions from the set of regression quantities. Regression is usually related, so it is impossible to determine the contribution of each regression quantity to the expected output energy. It is possible to transform the $p_{i}$ set into an orthogonal set by the OLS method, and then select those important regression quantities to determine the network has the best number of hidden layer nodes and the center of the hidden layer basis function. This was elaborated by Li et al. (2015).

The regression matrix $\mathrm{P}$ can be decomposed into

$$
P=W A
$$

Where $\mathrm{W}$ is an $\mathrm{N} \times \mathrm{N}$ orthogonal matrix, $W=\left[w_{1}, w_{2}, \ldots, w_{N}\right]$, and $W^{T} W=H, \mathrm{~A}$ is an $\mathrm{N} \times \mathrm{N}$ upper triangular matrix with a diagonal of 1 .

$$
A=\left[\begin{array}{ccccc}
1 & \alpha_{12} & \alpha_{13} & \cdots & \alpha_{1 N} \\
0 & 1 & \alpha_{23} & \cdots & \alpha_{2 N} \\
\vdots & \ddots & \ddots & \ddots & \vdots \\
\vdots & \ddots & \ddots & 1 & \alpha_{N-1 N} \\
0 & \cdots & \cdots & 0 & 1
\end{array}\right]
$$

So, equation (10) can be written as

$$
\begin{gathered}
Y=W G+E \\
G=\left[\begin{array}{ccc}
g_{11} & \cdots & g_{1 n 0} \\
\vdots & \ddots & \vdots \\
g_{N 1} & \cdots & g_{N n 0}
\end{array}\right] \\
\hat{G}=H^{-1} W^{T} Y
\end{gathered}
$$

And $\hat{\theta} \hat{G}$ are satisfied again.

$$
A \hat{\theta}=\hat{G}
$$


From the above formula, $\dot{\theta}$ can be easily solved.

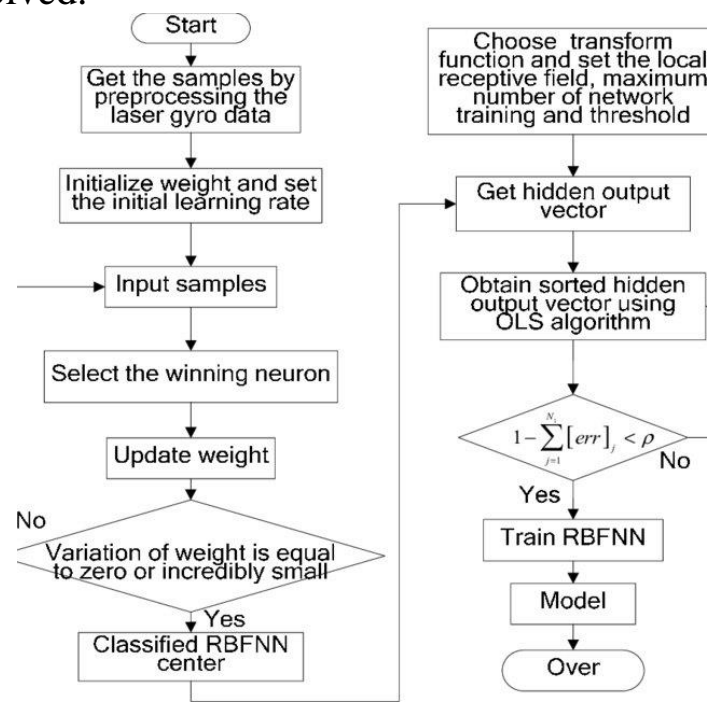

Fig. 2. Orthogonal least squares algorithm.

\section{Mathematical model and simulation of satellite attitude control system}

As shown in Fig. 1, the satellite attitude control system includes satellite attitude dynamics, kinematics, control module, and the actuator use a three-axis magnetic torque device, and thus includes a geomagnetic field model and a magnetic torque model. The control system obtains the attitude information directly from the attitude kinematics module.

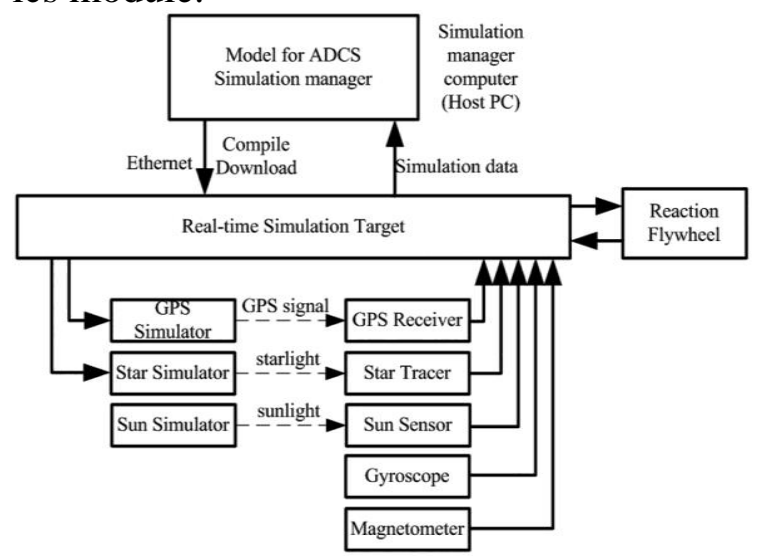

Fig. 3. Attitude simulation system structure.

\subsection{Coordinate system}

The attitude calculation of the satellite needs to involve a certain coordinate system. The coordinate system used in this model is the inertial coordinate system, the second orbital coordinate system and the satellite body coordinate system. The origins of the three coordinate systems are all at the geometric center of the satellite. The three axes of the inertial coordinate system point to a fixed direction (for example, the initial direction) of the space; the $\mathrm{z}$-axis of the second orbital coordinate system points to the center of the earth, the $\mathrm{X}$-axis is perpendicular to the z-axis, and the right-hand rule is along the direction of the satellite speed and the direction of the y-axis. According to Qishou et al. (2018), it is determined that the satellite ontology coordinate system coincides with the second orbital coordinate system in the initial state, and moves with the satellite body after the simulation starts, that is, it is fixed on the satellite.

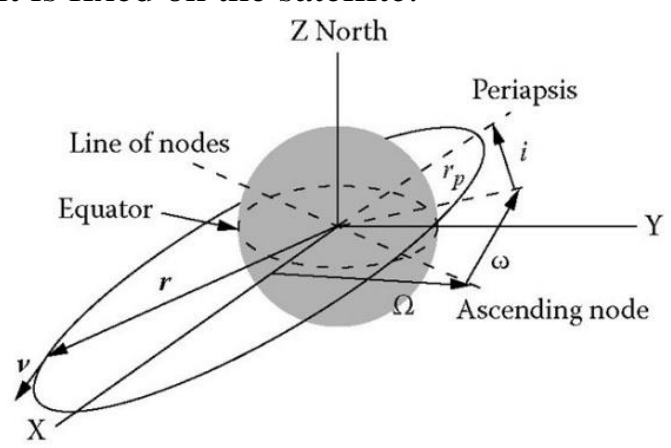

Fig. 4. Orbital coordinate system.

\subsection{Satellite attitude kinematics equation}

Considering that the satellite may have a large attitude change, the attitude quaternion is used to describe the attitude of the satellite. $\vec{\omega}_{o b}=\vec{\omega}_{i b}-\vec{\omega}_{0}\left(\vec{\omega}_{0}\right.$ is the satellite orbital angular 
velocity, $\vec{\omega}_{o b}$ is the projection of the angular velocity of the satellite relative to the orbital coordinate system in the ontology coordinate system, and $\omega_{b x}, \omega_{b y}, \omega_{b z}$ below is its component).

$$
\left(\begin{array}{l}
d q_{0} / d t \\
d q_{1} / d t \\
d q_{2} / d t \\
d q_{3} / d t
\end{array}\right)=\left(\begin{array}{cccc}
0 & -\omega_{b x} & -\omega_{b y} & -\omega_{b z} \\
\omega_{b x} & 0 & \omega_{b z} & -\omega_{b y} \\
\omega_{b y} & -\omega_{b z} & 0 & \omega_{b x} \\
\omega_{b z} & \omega_{b y} & -\omega_{b x} & 0
\end{array}\right)\left(\begin{array}{l}
q_{0} \\
q_{1} \\
q_{2} \\
q_{3}
\end{array}\right)
$$

\subsection{Satellite attitude data solution}

Numerical integration has a large error over a long period of time, so the following Euler method is chosen.

$$
\left\{\begin{array}{l}
u^{\prime}=f(t, u, v), u\left(t_{0}\right)=u_{0} \\
v^{\prime}=g(t, u, v), v\left(t_{0}\right)=v_{0}
\end{array}\right.
$$

For $\vec{J} \cdot \dot{\vec{\omega}}_{i b}+\vec{\omega}_{i b} \times \vec{J} \cdot \vec{\omega}_{i b}=\vec{T}$, it can be converted to $\dot{\vec{\omega}}_{i b}=\vec{J}^{-1} \cdot\left(\vec{T}-\vec{\omega}_{i b} \times \vec{J} \cdot \vec{\omega}_{i b}\right)$. In this way, then, $f=\vec{J}^{-1} \cdot\left(\vec{T}-\vec{\omega}_{i b} \times \vec{J} \cdot \vec{\omega}_{i b}\right)$, (torque $\mathrm{T}$ is also a variable related to the attitude of the satellite.)

$$
g=\left(\begin{array}{cccc}
0 & -\omega_{b x} & -\omega_{b y} & -\omega_{b z} \\
\omega_{b x} & 0 & \omega_{b z} & -\omega_{b y} \\
\omega_{b y} & -\omega_{b z} & 0 & \omega_{b x} \\
\omega_{b z} & \omega_{b y} & -\omega_{b x} & 0
\end{array}\right)\left(\begin{array}{c}
q_{0} \\
q_{1} \\
q_{2} \\
q_{3}
\end{array}\right)
$$

\subsection{Magnetic torque model}

The magnetic torque device uses the interaction between its own magnetic moment and the geomagnetic field to generate a moment acting on the star. Ignore other external magnetic fields that are unexpected in the geomagnetic field, then $\mathrm{T}$ is:

$$
\vec{T}=\vec{M} \times \vec{B}
$$

$\vec{M}$ is the magnetic moment vector of the magnetic moment, and $\vec{B}$ is the magnetic flux density vector (magnetic field strength) of the geomagnetic field.
The hollow magnetic moment device was selected as the modeling object in the modeling, which is mainly to simplify the model. Its magnetic moment size $|\vec{M}|$ :

$$
|\vec{M}|=A N I
$$

$A$ is the coil area, $\mathrm{N}$ is the number of turns of the coil, and I is the coil current. The direction of the magnetic moment is determined by the direction in which the magnetic torque device is mounted by Mehrjardi et al. (2015). If a three-axis magnetic torque device is installed, the size of each component is determined by equation (22).

Table 1

Gaussian coefficient of IGRF2005 model

\begin{tabular}{|c|c|c|c|c|c|}
\hline $\mathrm{N}$ & $\mathbf{M}$ & $g_{n}^{m}\left(T \times 10^{-9}\right)$ & $h_{n}^{m}\left(T \times 10^{-9}\right)$ & $\dot{\boldsymbol{s}}_{n}^{m}\left(T \times 10^{-9} / y\right)$ & $\dot{h}_{n}^{m}\left(T \times 10^{-9} / y\right)$ \\
\hline 1 & 0 & $\begin{array}{c}- \\
29556 . \\
8\end{array}$ & 0.0 & 8.8 & 0.0 \\
\hline 1 & 1 & $\begin{array}{c}- \\
1671.8\end{array}$ & 5080.0 & 10.8 & -21.3 \\
\hline 2 & 0 & - & 0.0 & -15.0 & 0.0 \\
\hline 2 & 1 & 3047.0 & -2594.9 & -6.9 & -23.3 \\
\hline 2 & 2 & 1656.9 & -516.7 & -1.0 & -14.0 \\
\hline 3 & 0 & 1335.7 & 0.0 & -0.3 & 0.0 \\
\hline 3 & 1 & - & -200.4 & -3.1 & 5.4 \\
\hline 3 & 2 & 1246.8 & 269.3 & -0.9 & -6.5 \\
\hline 3 & 3 & 674.4 & -524.5 & -6.8 & -2.0 \\
\hline
\end{tabular}

Table 2

Gaussian coefficient of WMM2005 model

\begin{tabular}{|c|c|c|c|c|c|}
\hline $\mathrm{N}$ & $\mathrm{M}$ & $g_{n}^{m}\left(T \times 10^{-9}\right)$ & $h_{n}^{m}\left(T \times 10^{-9}\right)$ & $\dot{s}_{n}^{m}\left(T \times 10^{-9} / y\right)$ & $\dot{h}_{n}^{m}\left(T \times 10^{-9} / y\right)$ \\
\hline 1 & 0 & $\begin{array}{c}- \\
29556 . \\
8\end{array}$ & 0.0 & 8.0 & 0.0 \\
\hline 1 & 1 & $\begin{array}{c}- \\
1671.7\end{array}$ & 5079.8 & 10.6 & -20.9 \\
\hline 2 & 0 & $\begin{array}{c}- \\
2340.6\end{array}$ & 0.0 & -15.1 & 0.0 \\
\hline
\end{tabular}




\begin{tabular}{|l|c|c|c|c|c|}
\hline 2 & 1 & 3046.9 & -2594.7 & -7.8 & -23.2 \\
\hline 2 & 2 & 1657.0 & -516.7 & -0.8 & -14.6 \\
\hline 3 & 0 & 1335.4 & 0.0 & 0.4 & 0.0 \\
\hline 3 & 1 & - & -199.9 & -2.6 & 5.0 \\
& & 2305.1 & & & \\
\hline 3 & 2 & 1246.7 & 269.3 & -1.2 & -7.0 \\
\hline 3 & 3 & 674.4 & -524.5 & -6.8 & -2.0 \\
\hline
\end{tabular}

\subsection{Mathematical simulation results}

The entire attitude control system was built in MATLAB/ Simulink as described above. Satellite can make a quality characteristic parameters like this: $J_{x}=16.0663, J_{y}=16.0368, J_{z}=0.1461$. Control law parameter: $\mathrm{K}=2$. Magnetic torque device parameters: select $\mathrm{A}=0.005, \mathrm{~N}=100, \mathrm{I}$ is simplified to be proportional to the input voltage, take $R=50$ initial condition setting: initial angular velocity: $0.086 \mathrm{rad} / \mathrm{s}$ (each angular velocity is $0.05 \mathrm{rad} / \mathrm{s}$ ); initial The three Euler angles are all 0 degrees; the simulation time is 20,000 seconds shown by Shaofei et al. (2019).

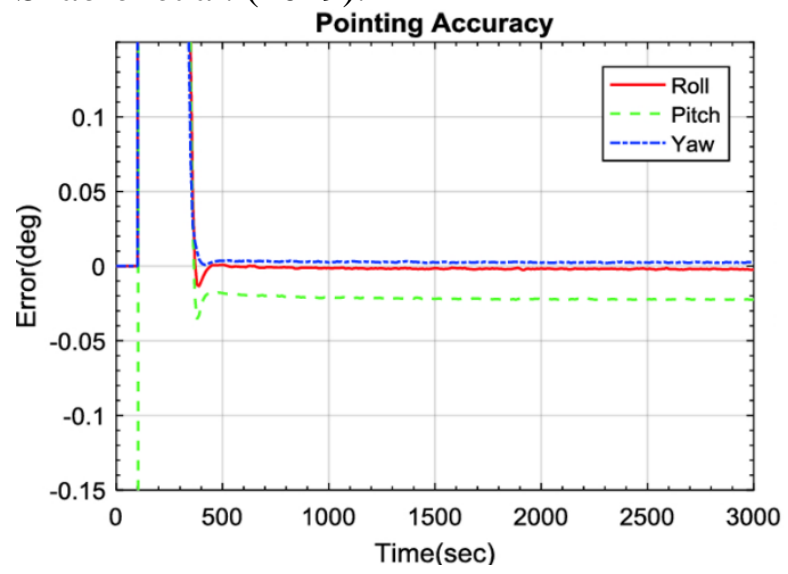

Fig. 5. Satellite attitude simulation accuracy.

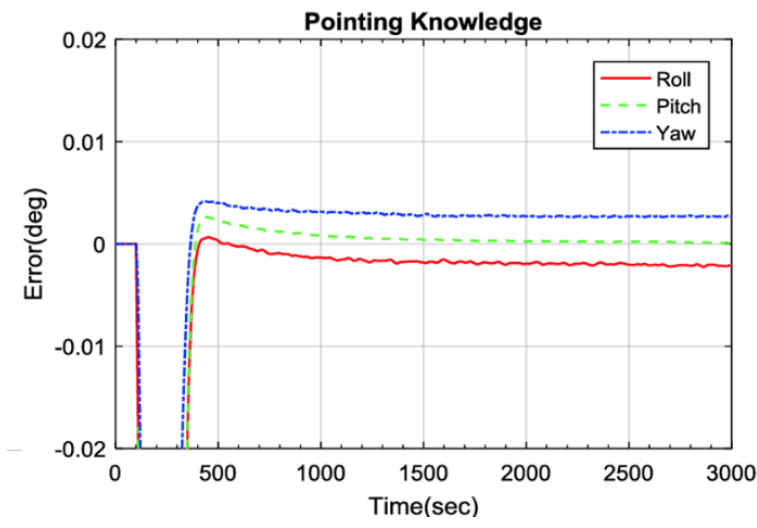

Fig. 6. Satellite attitude simulation pointing knowledge.

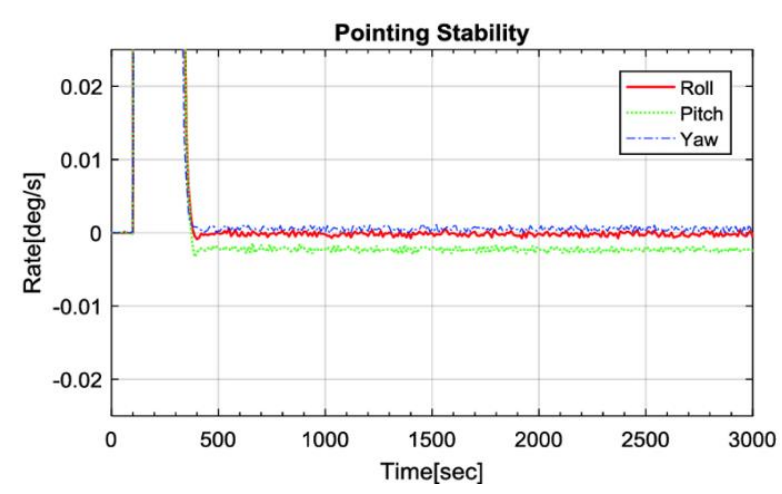

Fig. 7. Satellite attitude simulation stability.

Assuming that the sum of the other various disturbances is $T_{d y}=5 \times 10^{-4}[\cos (2 \pi t+0.5)+1] N^{\mathrm{o}} \mathrm{m}$, the system is mathematically simulated with and without compensation. The results are shown in Figure 7. The ordinates of the upper and lower figures are the satellite angle $\theta_{v}$ and the angular velocity $\omega_{y}$, respectively, and the abscissa is time $t$ as shown in Shaofei et al. (2019). Among them, the right picture shows the end of the simulation curve. It can be seen that the response speed of the system after the neural network disturbance compensation is significantly improved, and the steady-state error of the system is significantly reduced by Giri et al. (2017). 


\section{Conclusion}

This paper proposes an improved ROLS learning algorithm for RBF networks. Based on the algorithm, the neural network model of disturbance torque is established by using RBF network, and then the disturbance control neural network compensation control scheme of satellite attitude control system is designed. The mathematical simulation results show that the improved learning algorithm can effectively establish the RBF network model of the disturbance torque, and the proposed control scheme can improve the attitude control accuracy.

\section{References}

[1]Habibkhah, S., Arasi, J., \& Bolandi, H. Spacssim (2017), simulation and analysis software for mathematical modeling of satellite position and attitude control systems. Computing in Science \& Engineering, 19(5), 38-48.

[2]Steyn, W. H. Comparison of low-earthorbit satellite attitude controllers submitted to controllability constraints (2015), Journal of Guidance Control \& Dynamics, 17(4), 795-804.

[3]Fan, G. W., Chang, L., Dai, L., Kai, X. U., Yang, X. B., \& Amp, N (2015), Nonlinear model predictive control of agile satellite attitude maneuver. Optics \& Precision Engineering, 23(8) 2318-2327.

[4] Shaofei Wu (2020), Design of interactive digital media course teaching information query system, Information Systems and e-Business Management, 18(4), 793-807

[5]Shirazi, A., \& Mirshams, M (2016), Design and performance simulation of a satellite momentum exchange actuator. Aus- tralian Journal of Mechanical Engineering, 14(1), 1-9.

[6]Wang, S. Y., Min, L. I., Zhang, Y. C., Zhu, B. L., \& Hua-Yi, L. I (2015), Strong tracking unscented kalman filter algorithm based-on satellite attitude determination system. Electric Machines \& Control, 19(3)), 111-118.

[7] H. Li and Y. Man, "Relative radiometric calibration method based on linear CCD imaging the same region of non-uniform scene", International Symposium on Optoelectronic Technology and Application 2014: Optical Remote Sensing Technology and Applications, 2014. Available: 10.1117/12.2069564 [Accessed 26 October 2021].

[8] Qishou Ke,Shaofei Wu,Mingqing Wang,Yuntao Zou (2018) Evaluation of Developer Efficiency Based on Improved DEA Model, Wireless Personal Communications, 102(4), 3843-3849

[9] Mehrjardi, M. F., Sanusi, H., \& Mohd. Ali, M. A (2015), Three-axis attitude estimation of satellite through only twoaxis magnetometer observations using lkf algorithm. Metrology \& Measurement Systems, 22(4) , 577-590.

[10] Shaofei Wu, Jun Liu, Lizhi Liu (2019), Modeling method of internet public information data mining based on probabilistic topic model, The Journal of Supercomputing, 755,882-5897

[11] Shaofei Wu, Qian Zhang, Wenting Chen, Jun Liu, Lizhi Liiu (2019), Research on trend prediction of internet user intention understanding and public intelligence mining based on fractional differential method, Chaos, Solitons and Fractals, 128, 331-338

[12] Giri, D. K., \& Sinha, M ((2017), Finite-time continuous sliding mode magneto-coulombic satellite attitude control. 
IEEE Transactions on Aerospace \& Electronic Systems, 52(5) 2397-2412.

\section{Funding}

This project has not been funded by any organization or institute.

\section{Conflicts of Interest}

There is no conflict of interest with any co-authors or anyone.

\section{Availability of Data and Material}

Data is obtained using simulation results and it belongs to this work only.

\section{Code Availability}

\section{Author's Contribution}

First Author contributes design and implementation. Second Author collects the data and research gap analysis and so on.

\section{Ethics Approval}

The manuscript has not been submitted to any other journal. It follows all the ethics of the publishing

\section{Consent to participate}

We want to enhance our knowledge. We wanted to make people understand in precise form by the medium of paper.

\section{Consent for publication}

We want to publish our paper with no question of conflicts between both authors. 